August 4-7, 2013, Portland, Oregon, USA

DETC2013-12743

\title{
MANUFACTURE OF ARBITRARY CROSS-SECTION COMPOSITE HONEYCOMB CORES BASED ON ORIGAMI TECHNIQUES
}

\author{
Kazuya Saito * \\ Department of Mechanical and Biofunctional Systems \\ Institute of Industrial Science \\ the University of Tokyo \\ 4-6-1 Komaba, Tokyo 153-8505 \\ Japan \\ Email: saito-k@iis.u-tokyo.ac.jp
}

\author{
Sergio Pellegrino \\ California Institute of Technology \\ Pasadena, California 91125 \\ Taketoshi Nojima \\ Art Excel Co., Ltd \\ Hirakata, Osaka 573-1112 \\ Japan
}

\begin{abstract}
In recent years, as space structures have become large and require higher accuracy, composite honeycombs, which can reduce weight and have low thermal expansion, are in increasing demand. As observed in the design of antenna reflectors and rocket bodies, both flat and 3D-shaped cores are used in this field. However, these special honeycombs have high manufacturing costs and limited applications. This study illustrates a new strategy to fabricate arbitrary cross-section honeycombs with applications of advanced composite materials. These types of honeycombs are usually manufactured from normal flat honeycombs by curving or carving, but the proposed method enables us to construct objective shaped honeycombs directly. The authors first introduce the concept of the kirigami honeycomb, which is made from single flat sheets and has periodical slits resembling origami. In previous studies, honeycombs having various shapes were made using this method, and were realized by only changing folding line diagrams (FLDs). In this study, these 3D kirigami honeycombs are generalized by numerical parameters and fabricated using a newly proposed FLD design method, which enables us to draw the FLD of arbitrary cross-section honeycombs. Next, the authors describe a method of applying this technique to advanced composite materials. Applying the partially soft composite techniques, folding lines are materialized by silicon rubber hinges on carbon fiber reinforced plastic. Complex FLD patterns are then printed using masks on carbon fabrics. Finally, these foldable composites that are cured in corrugated shapes in
\end{abstract}

autoclaves are folded into honeycomb shapes, and some typical samples are shown with their FLDs.

\section{INTRODUCTION}

In the construction of aerospace components, lightweight, rigid, and strong honeycomb sandwich panels are required. In recent years, the use of composite materials has drastically increased in this field. In the case of sandwich panels, carbon fiber reinforced plastic (CFRP) face sheets are typically combined with an aluminum honeycomb. Currently, space structures are increasing in size and require greater degrees of accuracy; hence, the use of composites as a core material is a natural progression. It is promising for further reducing the weight of the body of satellites and enhancing the accuracy of their on-board equipment because of their low coefficients of thermal expansion (CTE). Furthermore, use of the same material for both the face sheet and the core prevents certain problems that would otherwise arise when combining materials that have different CTEs, such as CFRP-face aluminum honeycomb [1].

Various types of composite honeycombs [2-4] are commercially available, in addition to aluminum or nomex honeycombs. A comparable product is the CFRP honeycomb that has recently been used in antenna reflectors for high frequencies [5,6]. Other composites such as Kevlar honeycombs are also manufactured and used in floor panels of the latest airplane model. Quartz fiber, which has superior electrical properties has found the application in radome. 
Many studies on various types of core configurations that do not include hexagonal honeycombs have been reported. The main examples include lattice materials consisting of webs and struts [7-9]. Several configurations have already been proposed in this research area: octet-truss, the 2D and 3D Kagome structure, and the tetrahedral lattice. Square composite honeycombs, which are expected to show high in-plane stretching rigidity, have also been researched [10]. These square honeycombs are fabricated by assembling slotted rectangular composite sheets. The Z-fiber and X-Cor are truss structures that are fabricated by angled carbon fiber rods embedded in polymeric foam, and they are typical examples of composite sandwich panels [11] that have potential commercial applications.

However, these composite core materials are not regularly used in sandwich construction. Compared to standard aluminum or nomex honeycombs, their manufacturing costs are very high and they have limited applications. Another problem is difficulty of machining. In the manufacture of complex-shaped parts, the cores must have some degree of curvature. For aluminum honeycombs, this can be done using a contour cutter, a 3-D tracer, and numerically controlled machines. However, burrs and buckling of cell walls present a difficult problem for surface accuracy. It is clear that the machining of composite cores requires more expensive and sophisticated systems. Realizing curvatures in honeycombs is also difficult because they deform a saddle shape when bent. It requires special cell shapes such as flexcore [2] or cells having auxetic behavior $[12,13]$ for a large and accurate curvature.

This study proposes a novel method to construct arbitrary cross-section composite honeycombs. The basic idea originates from the fold-made paper honeycombs proposed by Nojima and Saito in 2007 [14], in which they attempted to apply origami and kirigami techniques to the creation of sandwich structures. Origami is the traditional Japanese art of paper folding and has received widespread attention from artists, architects, and mathematicians. Kirigami is a variation of origami. While traditional origami prohibits the cutting of paper, it is permissible in kirigami. Kirigami artists create remarkable patterns on paper using a combination of cutting and folding.

Figure 1 shows the basic concept of kirigami (or origami) honeycombs. The advantage of this method is that it can be extended to manufacture 3D (non-flat) honeycomb. This is achieved only by changing the folding line patterns. Some paper samples and their folding line diagrams (FLDs) are shown in Fig. 2. However, these previous studies [14] have not included non-convex-shaped cross-sections. Because of geometrical restrictions, their FLDs cannot be drawn on single sheets of flat paper. Generalized FLD design methods have also remained a challenge.

In this study, these 3D kirigami honeycombs are generalized by numerical parameters, and a new FLD design method is devised. This study also includes non-convex-shaped crosssections that have not been possible to realize in previous studies. The outline of the paper is as follows. First, the design method of the FLD is devised. This involves the calculation of the position of the folding lines and slits from given crosssectional shapes. The second part describes the condition pertaining to foldability and propose a modified method for unfoldable cross-sections. This approach enabled us to fold arbitrary cross-section honeycombs (including non-convex honeycombs) by folding single sheets. The third part describes a method of applying this technique to advanced composite materials. As an exapmle of kirigami composite honeycombs, a wingbox was already manufactured in autoclave-cured woven Kevlar fabric using the FLD around an airfoil profile[15]. In Ref.15, a dashed line cutting is used on a folding line. This study proposes a new method to introduce folding lines on CFRP; Silicon rubber is used as the matrix for hinge areas. Finally, these foldable composites that are cured in corrugated shapes in autoclaves are folded into honeycomb shapes, and some typical samples are shown with their FLDs.

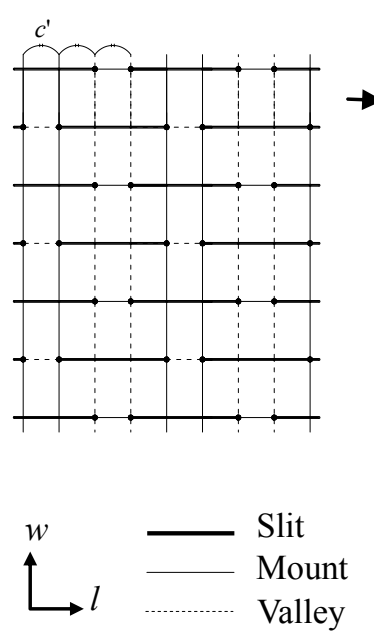

(a) (b)

(c)

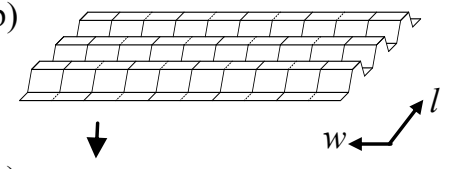

(d)
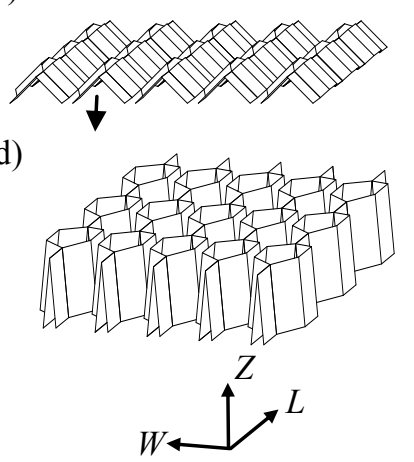

Fig. 1 Concept of kirigami honeycomb core. (a) Basic folding lines diagram. Thick lines: Slits. Fine lines: mountain folding lines. Dashed lines: valley folding lines. (b)-(d) The folding process for realizing a honeycomb shape.

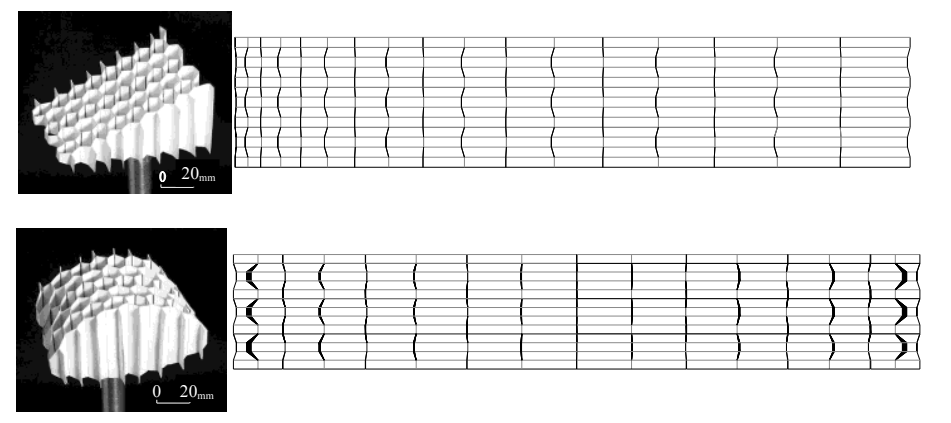

Fig. 2 Examples of 3D folded honeycombs and their folding line diagrams (FLDs). Upper: Tapered honeycomb. Lower: Convex curved honeycomb. Black lines and areas: Slits or cutouts. Gray lines: Folding lines. 


\section{NOMENCLATURE}

$\begin{array}{ll}C & \text { Cell size. } \\ c^{\prime} & \text { Gap length between } w \text {-direction folding lines. } \\ \theta & \text { Characteristic angle of honeycomb. } \\ X_{L} & \text { Honeycomb width for } L \text {-direction. } \\ X_{W} & \text { Honeycomb width for } W \text {-direction. } \\ N_{L} & \text { Number of cells for } L \text {-direction. } \\ N_{W} & \text { Number of cells for } W \text {-direction. } \\ \mathrm{A}_{i}, \mathrm{~B}_{i} & \text { FLD vertices. } \\ a_{i}, b_{i} & \text { FLD parameters. } \\ \mathrm{T}_{i}, \mathrm{U}_{i} & \text { Boundary line vertices. } \\ t_{i}, u_{i} & \text { Cross-section parameters. } \\ l_{i} & \text { Height of cell walls. } \\ s_{i} & \text { Widths of the slits. } \\ x_{l} & \text { FLD width for } L \text {-direction. }\end{array}$

\section{FOLDING LINE DIAGRAMS}

\section{Parameters for FLDs and Cross-Section Honeycombs}

This study considers the one-directional arbitrary cross-section honeycomb shown in Fig. 3. Here, the core thickness and curvature change only in the $W$ direction, which is the mechanical direction in commercial honeycombs. All cell walls are perpendicular to the $L W$ surface, and each cell has a regular hexagonal cross-section. Fig. 4 shows a representative FLD for a honeycomb such as the one shown in Fig. 3. First, we introduce new parameters that represent the position of vertices in FLDs and folded honeycomb shapes. Compared with the basic models shown in Fig. 1 (a), all folding lines that are parallel to the $w$ axis remain parallel and equally spaced, but the
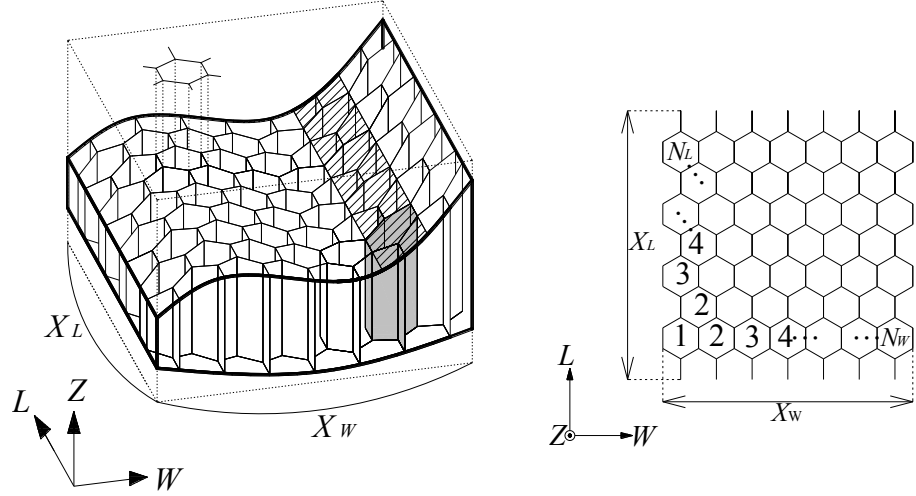

Fig. 3 One directional modified cross-section honeycomb. The core thickness and curvature change only in the $W$ direction. All cell walls are perpendicular to the $L W$ surface, and each cell has a regular hexagonal cross-section. position of the slits moves along the $w$ direction. To draw an FLD, the $w$ coordinate values of these slit vertices are required.

Considering periodicity and symmetry, a belt-shaped area is cut from the FLD and define the $l$ and $w$ axes. The representative vertexes $A_{0}, A_{1}, A_{2}, \ldots$ and $B_{0}, B_{1}, B_{2}, \ldots$ are chosen, as shown in Fig. 4. Each $w$ coordinate is defined as $a_{0}$, $a_{1}, a_{2}, \ldots$ and $b_{0}, b_{1}, b_{2}, \ldots$, which are called the FLD parameters. Meanwhile, parameters that represent the cross-sectional shape of the honeycombs are defined as follows. Fig. 5 shows how the vertices $\mathrm{A}_{i}$ and $\mathrm{B}_{i}$ in Fig. 3 are positioned in the $L W Z$ space after folding. Here, the built shape is projected onto the $W Z$ surface and the top and bottom boundary lines are defined as $\mathrm{T}_{0}, \mathrm{~T}_{1}$, $\mathrm{T}_{2} \ldots$ and $\mathrm{U}_{0}, \mathrm{U}_{1}, \mathrm{U}_{2}, \cdots$, as shown in Fig. 5. The cross-section parameters $t_{i}$ and $u_{i}$ are defined as their $Z$ coordinates.

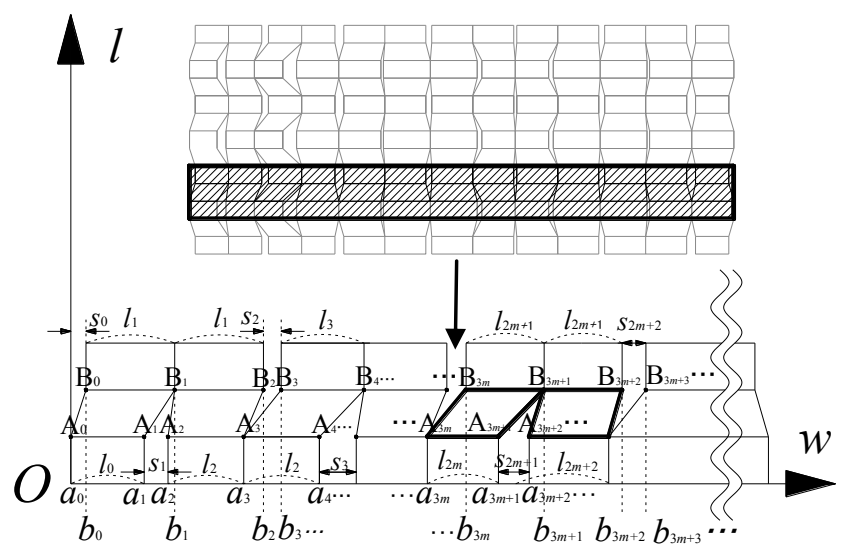

Fig. 4 Definition of the FLD parameters $a_{i}$ and $b_{i}$.
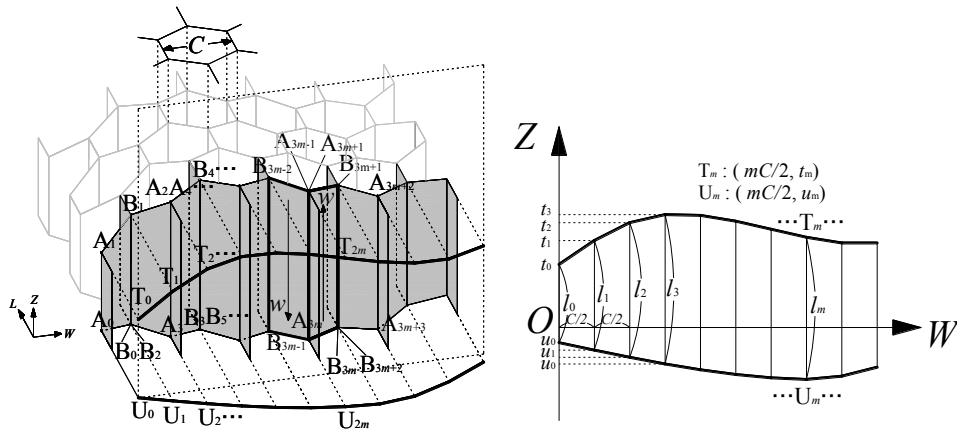

Fig. 5 Definition of the cross-section parameters $t_{i}$ and $u_{i}$. 


\section{Calculation of FLD Parameters}

In this section, a novel FLD design method is devised. This refers to the manner of calculating the FLD parameter $\left(a_{i}, b_{i}\right)$ from the given cross-section parameters $\left(t_{i}, u_{i}\right)$. First, the intervals between the slits that are represented by $l_{i}$ in Fig. 4 are defined as follows:

$$
\begin{aligned}
& l_{2 \mathrm{~m}}=a_{3 \mathrm{~m}+1}-a_{3 \mathrm{~m}}=a_{3 \mathrm{~m}}-a_{3 \mathrm{~m}-1} \\
& l_{2 \mathrm{~m}-1}=b_{3 \mathrm{~m}-1}-b_{3 \mathrm{~m}-2}=b_{3 \mathrm{~m}-2}-b_{3 \mathrm{~m}-3} \\
& \left(l_{0}=a_{1}-a_{0}, \mathrm{~m}=1,2,3 \cdots\right)
\end{aligned}
$$

The widths of the slits $s_{i}$ are also represented by $a_{i}$ and $b_{i}$.

$$
\begin{aligned}
& s_{2 \mathrm{~m}}=b_{3 \mathrm{~m}}-b_{3 \mathrm{~m}-1} \\
& s_{2 \mathrm{~m}-1}=a_{3 \mathrm{~m}-1}-a_{3 \mathrm{~m}-2}
\end{aligned}
$$

$$
\left(\mathrm{m}=1,2,3 \cdots, s_{0}=a_{0}-b_{0}\right)
$$

Using these equations, each FLD parameter can be represented as the sum of $l_{i}$ and $s_{i}$, as follows:

$$
a_{3 m}=\sum_{i=0}^{m-1}\left\{l_{2 i}+l_{2 i+2}+s_{2 i+1}\right\}
$$

Next, $l_{i}$ also corresponds to the cell thickness in fold-made honeycombs, as shown in Fig. 5, and can be represented by cross-section parameters.

$$
l_{i}=t_{i}-u_{i}
$$

In the FLD and its folded shape, each rectangle remains unchanged. A comparison of the solid-line rectangle in Figs. 4 and 5 therefore allows us to obtain the following equations about the relative positions of vertices.

$$
\begin{aligned}
& b_{3 \mathrm{~m}}-a_{3 \mathrm{~m}}=u_{2 \mathrm{~m}+1}-u_{2 \mathrm{~m}} \\
& b_{3 \mathrm{~m}-1}-a_{3 \mathrm{~m}}=u_{2 \mathrm{~m}}-u_{2 \mathrm{~m}-1}
\end{aligned}
$$

Using (7), (8), and (3), we can represent $s_{2 \mathrm{~m}}$ using cross-section parameters.

$$
\begin{aligned}
s_{2 \mathrm{~m}} & =\left(b_{3 \mathrm{~m}}-a_{3 \mathrm{~m}}\right)-\left(b_{3 \mathrm{~m}-1}-a_{3 \mathrm{~m}}\right) \\
& =u_{2 \mathrm{~m}+1}-2 u_{2 \mathrm{~m}}+u_{2 \mathrm{~m}-1}
\end{aligned}
$$

Using a similar process, $s_{2 \mathrm{~m}+1}$ is written in the form

$$
s_{2 \mathrm{~m}+1}=2 t_{2 \mathrm{~m}+1}-t_{2 \mathrm{~m}}-t_{2 \mathrm{~m}+2}
$$

Using (1)-(5), (8), and (9), the FLD parameter $a_{3 \mathrm{~m}}$ can be represented by a cross-section parameter as follows:

$$
a_{3 m}=\sum_{i=0}^{m-1}\left\{2 t_{2 i+1}-u_{2 i}-u_{2 i+2}\right\}
$$

We can also calculate other diagram parameters $a_{3 \mathrm{~m}+1}, a_{3 \mathrm{~m}-1}$ from (11);

$$
\begin{aligned}
& a_{3 m+1}=a_{3 m}+l_{2 m}=\sum_{i=0}^{m-1}\left\{2 t_{2 i+1}-2 u_{2 i+2}\right\}-u_{0}+t_{2 m} \\
& a_{3 m-1}=a_{3 m}-l_{2 m}=\sum_{i=0}^{m-1}\left\{2 t_{2 i+1}-2 u_{2 i}\right\}+u_{0}-t_{2 m}
\end{aligned}
$$

$b_{3 \mathrm{~m}}$ is provided from (7) and (11) as follow,

$$
b_{3 m}=\sum_{i=0}^{m-1}\left\{2 t_{2 i+1}-2 u_{2 i+2}\right\}-u_{0}+u_{2 m+1}
$$

then,

$$
\begin{gathered}
b_{3 m+1}=b_{3 m}+l_{2 m+1}=\sum_{i=0}^{m-1}\left\{2 t_{2 i+1}-2 u_{2 i+2}\right\}-u_{0}+t_{2 m+1} \\
b_{3 m+2}=b_{3 m}+2 l_{2 m+1}=\sum_{i=0}^{m-1}\left\{2 t_{2 i+1}-2 u_{2 i+2}\right\}-u_{0}-u_{2 m+1}+2 t_{2 m+1}
\end{gathered}
$$

These equations enable us to calculate FLD parameters from given cross-sectional shapes. However, it does not mean that we always can draw an FLD on a single flat paper. This would be possible only when all slits have positive widths. Fig. 6 shows the problem of the negative slits. As shown in Fig. 6 (a), the unit cell in normal flat honeycombs is folded from a paper with straight slits. Fig. 6 (b) shows a unit cell of the tapered honeycomb whose thickness increases linearly. Here, the widths of the slits equal to zero, as well as the flat unit cells. As shown in Fig. 6 (c), if the cell thickness changes with convex curves, the slit widths have positive values. This means that some cutouts are required on the paper. As shown in Fig. 2, the FLDs that consist of these types of unit cells can be constructed relatively easily, and various paper samples have been created in previous studies. However, when we constructed the unit cells as shown in Fig. 6 (d), which appear in non-convex curved honeycombs, the total angles surrounded a slit edge (vertices B and $\mathrm{E}$ in Fig. 6) over $2 \pi$. This implies that the slit width has a negative value and the papers overlap at the slits. It is therefore impossible to fold this type of unit cell from a single paper. This is the reason why the fabrication of non-convex curved honeycombs has not yet been realized in origami based methods. 


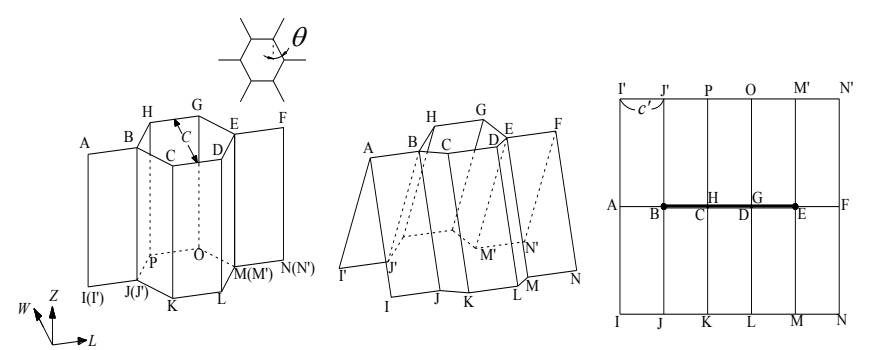

(a)

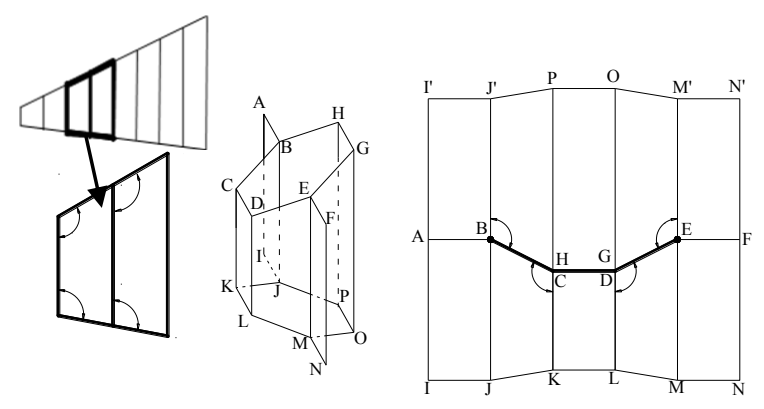

(b)
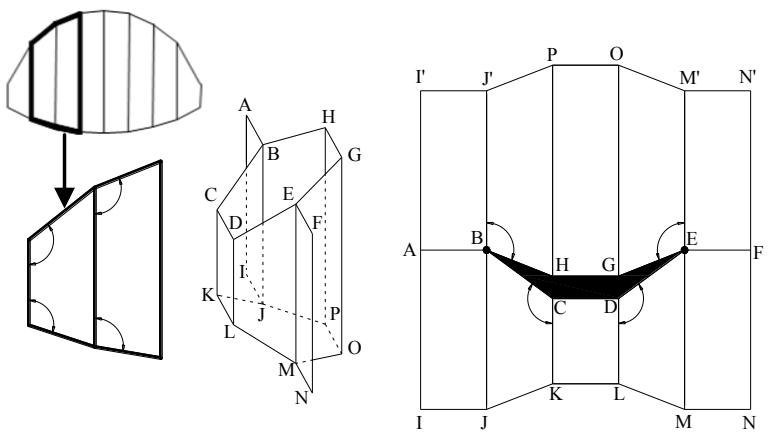

(c)
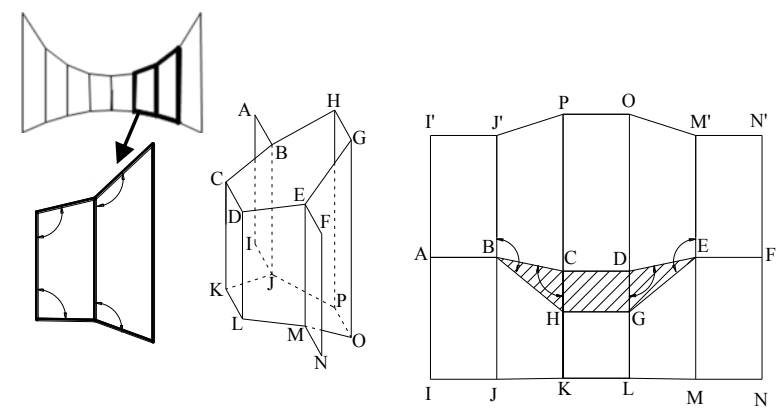

(d)

Fig. 6 Types of unit cells and their slit shapes. (a) Flat cell with straight slits. (b) Tapered cell with zero width slit. (c) Convex curved cell with positive width slit. (Hexagon EDCBHG is a cutout area.) (d) Non-convex curved cell with negative width cell. (Hexagon EDCBHG is an overlapping area.)

\section{Modified Method for Unfoldable Honey-combs}

This section is about the shape modifying method that avoids the negative-slit problem. Using (9) and (10), the conditions for foldable cross-sections $\left(s_{i} \geqq 0\right)$ can be written as follows:

$$
\begin{aligned}
& u_{2 \mathrm{~m}+1}+u_{2 \mathrm{~m}-1}-2 u_{2 \mathrm{~m}} \geqq 0 \\
& 2 t_{2 \mathrm{~m}+1}-t_{2 \mathrm{~m}+2}-t_{2 \mathrm{~m}} \geqq 0
\end{aligned}
$$

Normally, cross-sectional shapes are given as top and bottom boundary lines. In previous methods, these lines are divided for each $C / 2$ and approximated line segments, as shown in Fig. 7 (a). Here, $C$ is the call size. In this case, all cross-section parameters are defined individually; hence, the conditions of (17) and (18) are maintained in limited cases such as tapered or convex curved honeycombs.

In the suggested methods, boundary lines are divided for each $C$, as shown in Fig. 7 (b). Here, only even-numbered $\mathrm{T}_{i}$ and odd-numbered $\mathrm{U}_{i}$ are set on the boundary lines.

$$
\begin{aligned}
& t_{2 \mathrm{~m}}=f(m C) \\
& u_{2 \mathrm{~m}+1}=g((2 \mathrm{~m}+1) C / 2)
\end{aligned}
$$

Next, the remaining $\mathrm{T}_{2 m+1}$ and $\mathrm{U}_{2 m}$ are set on the mid-points of points that are before and after $\mathrm{T}$ and $\mathrm{U}$, as shown by the followed equations:

$$
\begin{aligned}
& t_{2 \mathrm{~m}+1}=\left(t_{2 \mathrm{~m}}+t_{2 \mathrm{~m}+2}\right) / 2 \\
& u_{2 \mathrm{~m}}=\left(u_{2 \mathrm{~m}-1}+u_{2 \mathrm{~m}+1}\right) / 2
\end{aligned}
$$

From (17) and (18), it is then confirmed that the slit widths $s_{i}$ are always equal to zero. This method permits to modify

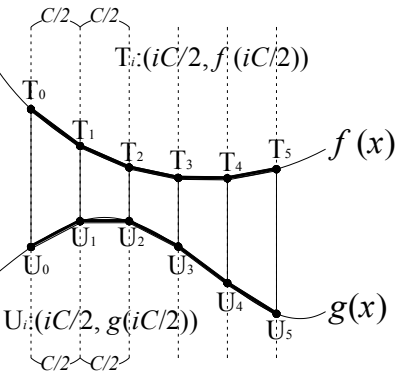

(a)

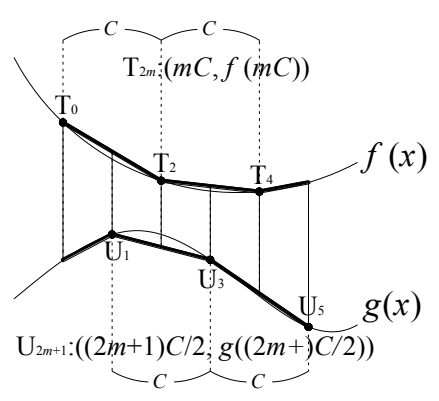

(b)
Fig. 7 Approximated cross sections. (a) Partitioning with $C / 2$ (Previous methods). (b) Partitioning with $C$ (Proposed method to modify the unfoldable case). 
unfoldable cross-sections and draw their FLDs on single flat sheets. This means that it becomes possible to fold arbitrary cross-section honeycombs from a single paper. Fig. 8 shows a sample sheet of paper folded into a honeycomb with parabolic surface and a sine curved surface. The specific steps of designing the FLD are shown in ANNEX.

\section{APPLICATION OF ADVANCED COMPOSITE MATERIALS}

\section{Folding lines on CFRP Sheets}

Using the above-mentioned methods, we can draw FLDs of arbitrary cross-section honeycombs. For paper and metallic honeycombs, it is not difficult to apply this method for mass production because there are various ways of bending and cutting them automatically. This section discusses the application of the method to advanced composite materials in aerospace applications.

With respect to the manufacturing process (Fig. 1), the challenge has been to find a method of introducing folding lines on a CFRP sheet. If a damage on a folding line is permitted, folding a thin composite sheet is simplicity itself. As commonly used in the paper craft techniques, scratching or dashed-line cutting on crease lines is effective to make accurate foldinglines. Taking a similar approach, an aerofoil shaped honeycomb are made from Kevlar sheets [15]. However, this method includes fiber breakages on folding lines and can be used only for one-time folding. In order to combine origami and composites, it is desired that folding lines are protected by some kind of soft materials. In examples of such techniques, some researchers have reported partially flexible composites that can be bent at soft-matrix areas [16,17]. Epoxy and silicon matrixes are used on the hard and soft parts, respectively. This study uses

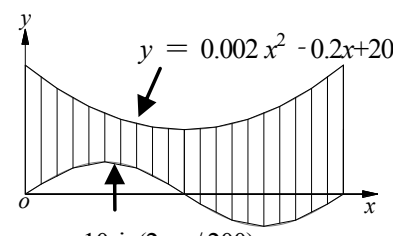

$y=10 \sin (2 \pi x / 200)$

(a)

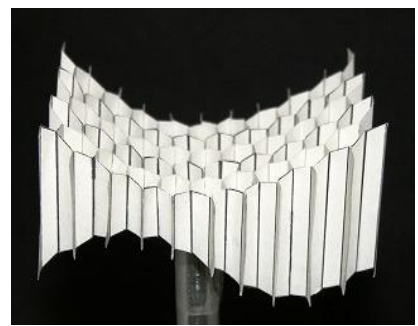

(b)

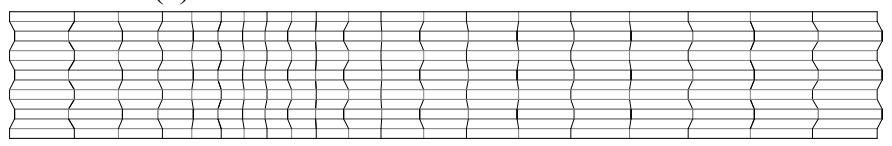

(c)

Fig.8 Honeycomb core with parabolic surface $(y=0.002$ $\left.x^{2}-0.2 x+20\right)$ and sine curved surface $(y=10 \sin (2 \pi x /$ 200)). (a) Cross section. (b) Paper sample ( $C=20 \mathrm{~mm}, 100$ $\times 200 \mathrm{~mm}$ ). (c) Development chart with $a_{i}$ and $b_{i}$. the mask to print more complex patterns of two types of matrices on carbon fabrics. Fig. 9 illustrates the concept of the mask method, which involves constructing a mask which has the same pattern as that of the FLD. During the resin infusion, it is put on a reinforcement sheet and the area of the folding lines is kept dry. After curing the epoxy, these dry areas are covered by a soft matrix. By using this techniques, we can freely draw folding patterns by only changing the mask patterns; This make it possible to fold origami by composite sheets. Figure 10 shows the process of making the composite crane which is known as traditional origami motif in Japan.
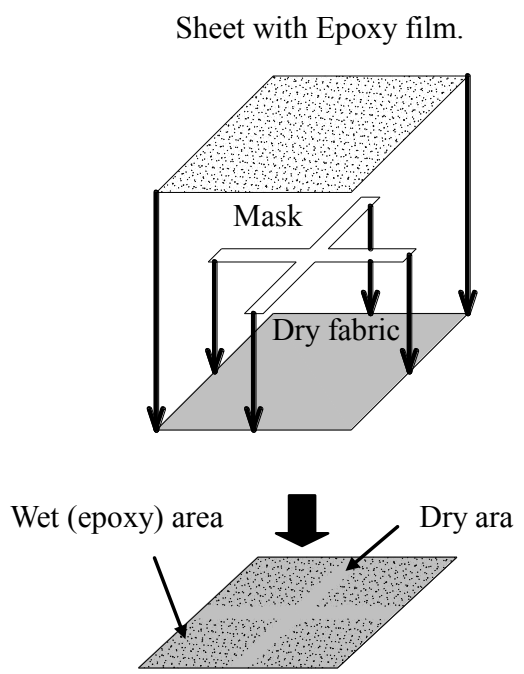

Fig.9 The concept of the mask method.

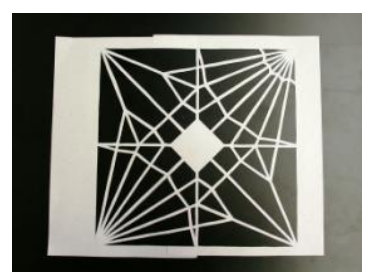

(a)

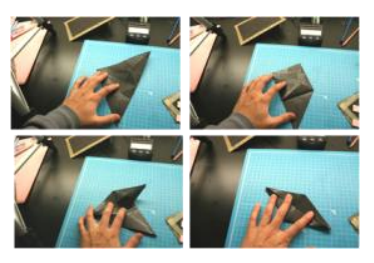

(c)

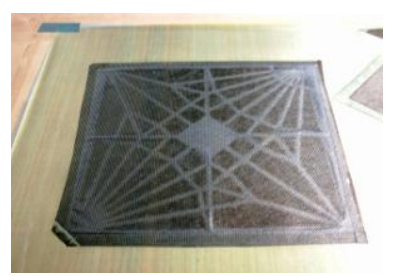

(b)

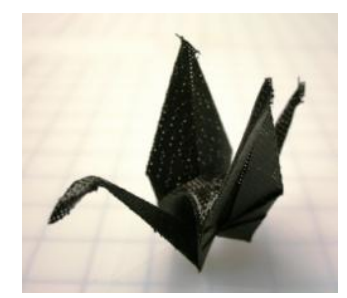

(d)
Fig.10 Composite origami crane. (a) Mask created from unfolded crease pattern of origami crane. (b) The partially soft composite sheets with the crane pattern. (c) Fold the sheets according to the folding process. (d) The folded shape. 


\section{CFRP Kirigami Honeycombs}

This study uses normal plain-woven carbon fabric G08017-1020 (HEXCEL Co., Ltd.) for reinforcement, normal epoxy resin Hex Ply 913 (HEXCEL Co., Ltd.) for the hard matrix, and silicon rubber CF19 -2615 (NuSil Technology Co., Ltd) for the soft matrix at the folding lines. Using the FLD design method, masks are constructed for various cross-section honeycombs. Fig. 11 (a) shows an example of a mask of a tapered honeycomb. Compared with FLDs on paper, the w-direction folding lines can be omitted because they are folded by a mold, and only slits and $l$-direction folding lines are reflected with no distinction. In a flat honeycomb, the FLD consists of straightlines; hence, straight masking tape can be used, as shown in Fig. 11 (b). By using these masks, the necessary folding lines are kept dry after resin infusion. These partially infused fabrics are cured into corrugated shapes on the molds. This study use aluminum trapezium rods that are obtained by cutting the hex rods in half, as shown in Fig. 12. The size of the rods corresponds to the cell size of folded honeycombs. Fig. 13 shows a sketch of the lay-up. The fabric is sanded with the release films and additional hex rods are used for holding. These partially resin infused sheets are cured at $125^{\circ} \mathrm{C}$ and 700 $\mathrm{kN} / \mathrm{m}^{2}$ for $60 \mathrm{~min}$ in the autoclave. Fig. 14 shows the sample after curing. The gray stripes that are seen are the dry areas. Silicon rubber is used to coat these areas and cured at $150^{\circ} \mathrm{C}$ for $30 \mathrm{~min}$. At this point, the cured silicon covers the slit lines as well as the folding lines. We therefore have to cut them according to the FLD. Fig. 15 (a) shows this process. After introducing slits, they can be folded into honeycomb shapes, as

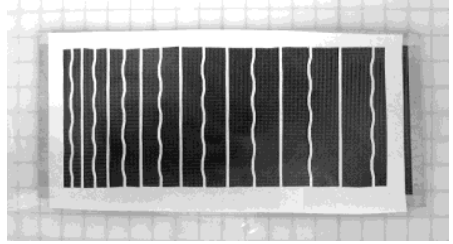

(a)

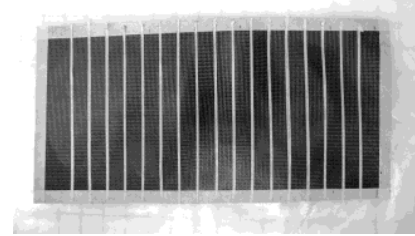

(b)
Fig.11 Samples of the masks. (a) Tapered honeycomb. (b) Flat honeycomb.

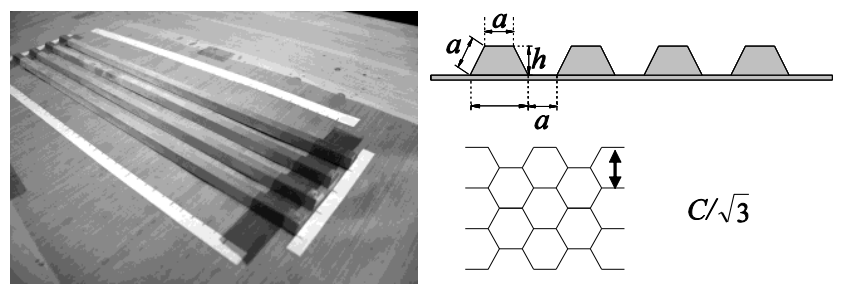

Fig. 12 The mold is made from $450 \mathrm{~mm}$ length aluminum rods $(c=14.3 \mathrm{~mm}(9 / 16 \mathrm{in}), h=7.15 \mathrm{~mm} 、 \quad a=8.25$ $\mathrm{mm})$. shown in Fig. 15 (b). Commercial acrylic adhesive is used to fix their folded shapes. Fig. 16 shows comprehension CFRP samples and their original FLDs. Figs. 16 (a) and (b) show the tapered honeycomb and the aerofoil (NACA2415) honeycombs, respectively. It is possible to construct curved cores that include non-convex cross-sections, as shown in Fig. 16 (c).

\section{Discussion}

This study used masks to infuse two types of matrix on single CFRP sheets, and we first put epoxy on dry fabrics. However, there are various methods for realizing folding lines

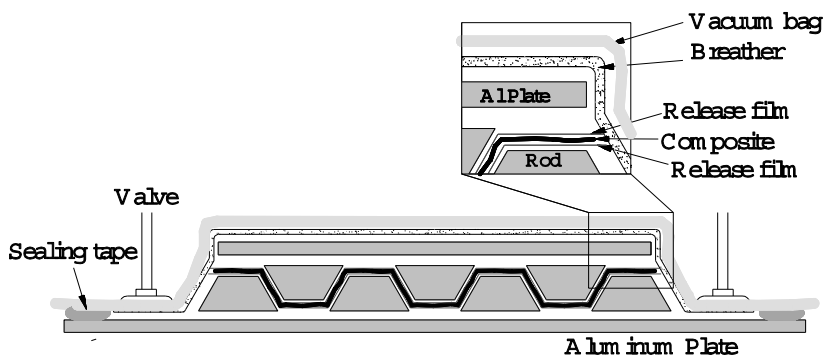

Fig. 13 Schematic illustration of the lay-up.

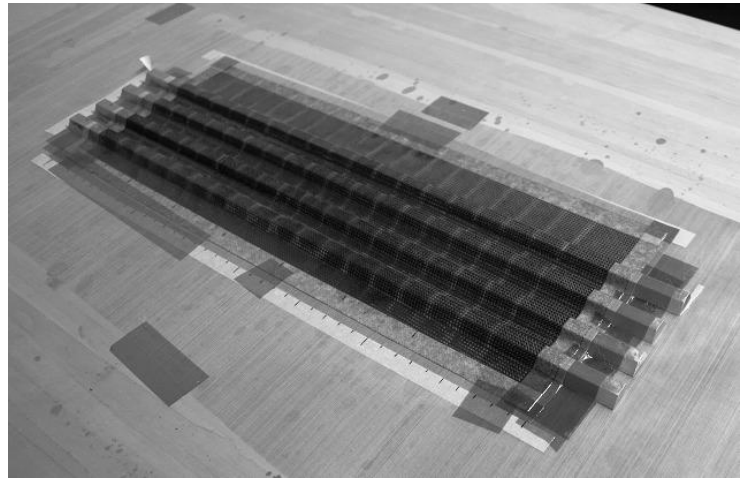

Fig. 14 Cured CFRP sheets.

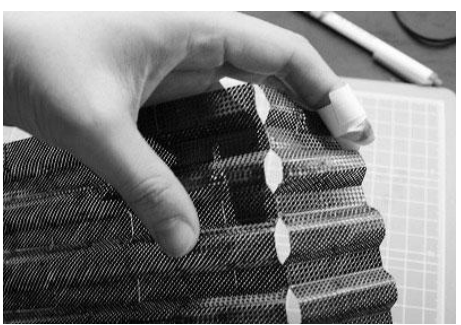

(a)

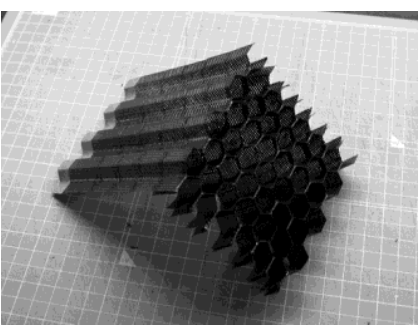

(b)
Fig. 15 (a) Corrugated CFRP sheet with slits and folding lines and (b) its folding process. 

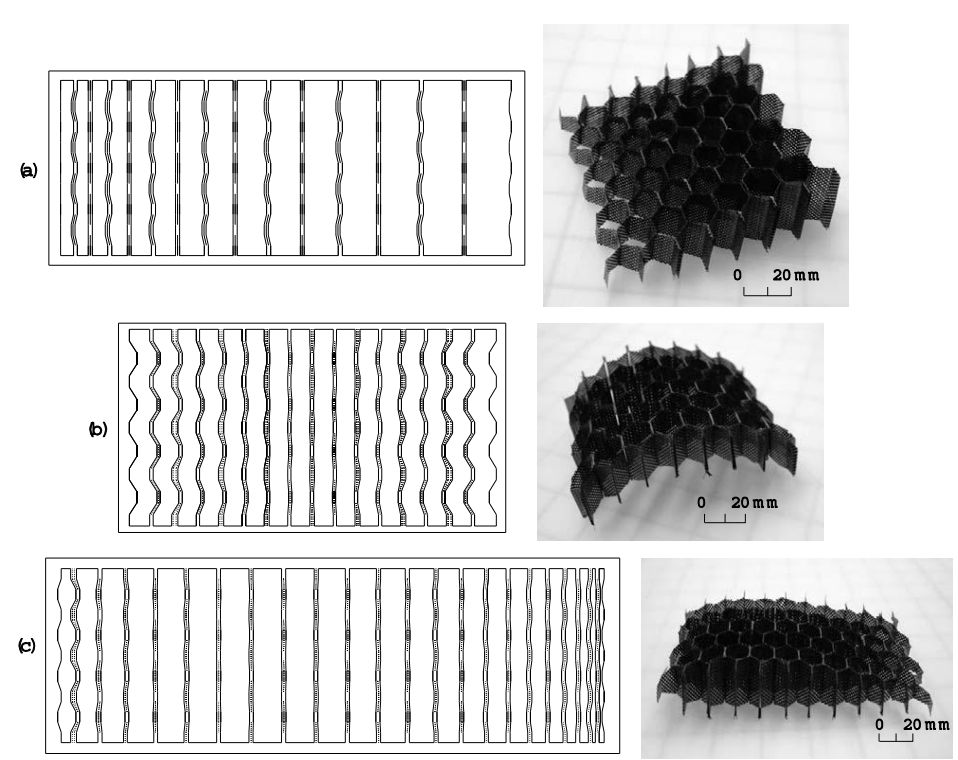

Fig. 16 Samples of CFRP 3D honeycomb cores and their mask patterns. (a) Tapered core $(C=14.3[\mathrm{~mm}])$. (b) Curved core $(C=14.3[\mathrm{~mm}])$. (c) Aerofoil $(C=14.3$ $[\mathrm{mm}])$.

on CFRP sheets. For example, it is possible to cover the folding lines with silicon rubber. Instead of using masks, stamps or printer systems can be used to infuse silicon rubber. There are several choices according to the types of fabrics and matrices. As mentioned before, another option is to partially cut (perforate) the reinforcements. An automated cutting machine is used to cut prepreg sheets and some samples have already been made with this method. In addition, this study is not limited to regular hexagonal honeycombs. By only changing the shape of the rods in Fig. 12, other cell configurations, including over expanding (OX) cells, can be manufactured, and a related study about negative Poisson's ratio (NPR) honeycomb has already begun.

This method approximates a cross section by continuing line segments, and it might cause the decrease of surface accuracy especially in large curvature. However, selecting small cell size can avoid this problem. Usually, the cell size is enough small comparing to curvatures of whole structures in aerospace components. About mechanical properties such as shear rigidity and compressive stiffness which are mainly required for cores, kirigami honeycomb is thought to have equivalent performance of current major honeycomb core if we select same materials, foil thickness, cell size and adhesive. In addition, foils are connected each other in kirigami honeycombs while they are separated in major honeycomb. It may provide higher structural strength to kirigami honeycombs.

\section{CONCLUSION}

By folding a thin flat sheet with periodical slits, various cross-section honeycombs were manufactured on the basis of origami and kirigami techniques. This study reveals the geometric relationship between an FLD and a cross-section of a folded honeycomb. They are represented by numerical parameters and fabricated using a newly proposed FLD design method. In addition, the foldability of 3D honeycombs was discussed, and we proposed a modified method for dealing with unfoldable cases. The above achievements make it possible to fold one directional arbitrary cross-section honeycombs from single flat sheets. Next, this study applies a kirigami honeycomb to advanced composite materials. Folding lines are materialized by soft-matrix (silicon rubber) hinges, and the mask method is devised to control the matrix areas on fabrics.

One of the major advantages of the proposed method is that we can directly manufacture arbitrary cross-section honeycombs without the need for difficult and expensive processing, which is normally required. The automation of the folding and gluing processes remains a challenge, but drastic cost reductions can be achieved when this method is adapted for mass production because of its versatility. This study also has significant potential for the continuous production of honeycombs.

\section{ACKNOWLEDGMENTS}

This work has been supported by Grant-in-Aid for JSPS Fellows (19-5987) by the Japan Society for Promotion of Sciences.

\section{REFERENCES}

[1] Thomsen, O.T., Manks, W.M., "An improved model for the prediction of intra-cell buckling in CFRP sandwich panels under in-plane compressive loading”, Composite Structures. 2004; 65, pp.259-268.

[2] Ultracor, Inc., http://www.ultracorinc.com

[3] Core composite, Inc., http://www.corecomposites.com

[4] JPS composite materials, Inc., http://jpsglass.com

[5] Pfeiffer, E.K., et.al. "Highly Stable Antenna Structure Technologies". In: Proceedings of 32nd ESA Antenna Workshop on Antennas for Space Applications. Noordwijk, October, 2010.

[6] Yarza, A., Castro, O., Prowald, J.S., and Liminana, C., "High stability large reflectors for $\mathrm{Ka}$ Band". In: Proceedings of European Conference on Antennas and Propagation. Barcelona, April, 2010.

[7] Wicks, N., and Hutchinson, J.W., "Optimal Truss Plates". Journal of Solids and Structures, 2001; 38: 5183-6165.

[8] Wadley, H.N.G., Fleck, N.A., Evans, A.G., 2003, "Fabrication and structural performance of periodic cellular metal sandwich structures". Composites Science and Technology, 63(16), pp. 2331-2343. 
[9] Finnegan, K., Kooistra, G., Wadley, H.N.G., Deshpande, V.S., 2007 "The Compressive Response of Carbon Fiber Composite Pyramidal Truss Sandwich Cores". International Journal of Materials Research, 12, pp.12641272.

[10] Russell, B.P., Deshpande, V.S., and Wadley, H.N.G., 2008, "Quasistatic Deformation and Failure Modes of Composite Square Honeycombs", Journal of Mechanics of Materials and Structures, 3(7), pp.1315-1340.

[11] Marasco, A.I., Cartié, D.D.R., Partridge, I.K., Rezai, A., 2006, "Mechanical properties balance in novel Z-pinned sandwich panels: Out-of-plane properties". Composites Part A: Applied Science and Manufacturing, 37(2): 295302.

[12] Evans, K.E., 1991, "Design of Doubly Curved Sandwich Panels with Honeycomb Cores". Composite Structures, 17, pp.95-111.

[13] Scarpa F, Jacobs S, Coconnier C, Toso M, DiMaio D., 2010, "Auxetic shape memory alloy cellular structures for deployable satellite antennas: design, manufacture and testing". In Proceedings of the 14th International Conference on Experimental Mechanics.

[14] Nojima, T., and Saito, K., 2006, "Development of UltraLight Core Structure", JSME International A, 49(1), pp.38.

[15] Saito, K., Agnese, F., and Scarpa, F., , "A Cellular Kirigami Morphing Wingbox Concept”, 2011, Journal of Intelligent Material Systems and Structures, 22, pp.935-944.

[16]Lopez, J.F., and Pellegrino, S., 2009, "Folding of thinwalled composite structures with a soft matrix", In Proceedings of 50th AIAA/ASME/ASCE/AHS/ASC Structures, Structural Dynamics and Materials Conference. Palm Springs, May, 2009.

[17] Todoroki, A., Kumagai, K., and Matsuzaki, R., 2009, "Selfdeployable Space Structure using Partially Flexible CFRP with SMA Wires". Journal of Intelligent Material Systems and Structures., 20, pp.1415-1424.

\section{ANNEX}

\section{DESIGN PROCESS FOR THR FOLDING LINE DIAGRAM}

This annex describes the specific steps for design process for FLD by using the example of Fig. 8 model. First, trace the cross-section of the target honeycomb on $x-y$ surface. Define the right and left boundary as $x=0, x=X_{w}$, express the top and bottom boundary with two curves $y=\mathrm{f}(x), y=\mathrm{g}(x)$ (Fig. A-1(a)). This example uses following a parabolic curve and a sine curve.

$$
\begin{aligned}
& f(x)=0.002 x^{2}-0.2 x+20 \\
& g(x)=10 \sin (2 \pi x / 200)
\end{aligned}
$$

Cell size $C$, honeycomb width for $w$-direction $X_{w}$, and the number of cell for w-direction $N_{\mathrm{w}}$ are defined by using following relation.

$$
C=X_{w} / N
$$

Here, we choice $X_{w}=200, N_{W}=10$, and $C=20$. The Gap length between $l$-direction folding lines $c^{\prime}$ (see Fig. A-2(a)) is determined as follow,

$$
c^{\prime}=C /(2 \cos \theta)
$$

The characteristic angle $\theta$ is shown in Fig.6(a). In a regular hexagonal honeycomb, $\theta$ is equals to $\pi / 6$, so $c^{\prime}=11.5$.
Secondly, divide the cross section into $2 N_{W}$ trapeziums for each $C / 2$ as shown in Fig. A-1(b). Then, vertexes on upper boundary are defined as $\mathrm{T}_{i}$, and lower as $\mathrm{U}_{i}$. If the target crosssection meet the foldable condition given by (17) and (18), the $\mathrm{y}$-coordinate of $\mathrm{T}_{i}$ and $\mathrm{U}_{i}$ can be used as cross-section parameters $t_{i}$ and $u_{i}$. Otherwise, it is necessary to modify the vertexes position according to (21)(22); remove the oddnumbered upper vertexes and even-numbered lower vertexes on the mid-points of points that are before and after $T$ and $U$ as shown in Fig.A-1(c). Table A-1 shows these modified crosssection parameters. The terms marked $*$ express the modified vertexes. The other terms express the vertexes on the curves of $y=\mathrm{f}(x)$ and $y=\mathrm{g}(x)$.

Thirdly, Put $t_{i}, u_{i}$ of Table A-1 in the equation (11) (16), calculate the FDL parameters $a_{i}$ and $b_{i}$. Table A-2 shows the results. In Table A-2, $a_{3 m+1}$ and $a_{3 m+2}$ equal to $b_{3 m+2}$ and $b_{3 m+3}$ respectively $(\mathrm{m}=0,1,2 \cdots 9)$. This is because after the above modification, all slit widths become zero, so $\mathrm{A}_{3 m+1}$ and $\mathrm{A}_{3 m+2}$ are unified with $\mathrm{B}_{3 m+2}$ and $\mathrm{B}_{3 m+3}$ respectively.

Finally, construct the belt-like partially FLD as shown in Fig.A-2(a) by using Table A-2. Repeating and reflection of this drawing gives the whole FLD. By marking the each of the three parts of Fig.A-2(a) with $\mathbf{A}, \mathbf{B}$, and $\mathbf{C}$, this repetition pattern express as $[\mathbf{A B C} \mathbf{C}][\mathbf{A B C} \mathbf{C}] \cdots[\mathbf{A B C} \mathbf{C}] \mathbf{A}$ as shown in Fig.A2 . Here, $\mathbf{a}$ expresses the reversed image of $\mathbf{B}$. The $l$-directional length of FLD is determined by the number of the L-direction cells and expressed by follow.

$$
x_{l}=\left(2 N_{L}+3\right) c^{\prime}
$$


Table A-1 Cross section parameters of Fig. 15 (mm).

\begin{tabular}{rrr}
$i$ & \multicolumn{1}{c}{$t_{i}$} & \multicolumn{1}{c}{$u_{i}$} \\
\hline 0 & 40.0 & 0.00 \\
\hline 1 & $* 36.4$ & 3.09 \\
\hline 2 & 32.8 & $* 5.59$ \\
\hline 3 & $* 30.0$ & 8.09 \\
\hline 4 & 27.2 & $* 9.05$ \\
\hline 5 & $* 25.2$ & 10.0 \\
\hline 6 & 23.2 & $* 9.05$ \\
\hline 7 & $* 22.0$ & 8.09 \\
\hline 8 & 20.8 & $* 5.59$ \\
\hline 9 & $* 20.4$ & 3.09 \\
\hline 10 & 20.0 & $* 0.00$ \\
\hline 11 & $* 20.4$ & -3.09 \\
\hline 12 & 20.8 & $*-5.59$ \\
\hline 13 & $* 22.0$ & -8.09 \\
\hline 14 & 23.2 & $*-9.04$ \\
\hline 15 & $* 25.2$ & -10.0 \\
\hline 16 & 27.2 & $*-9.05$ \\
\hline 17 & $* 30.0$ & 8.09 \\
\hline 18 & 32.8 & $* 5.59$ \\
\hline 19 & $* 36.4$ & 3.09 \\
\hline 20 & 40.0 & 0.00 \\
\hline
\end{tabular}

(a)

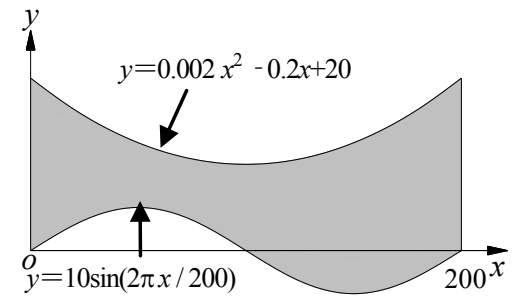

(b)

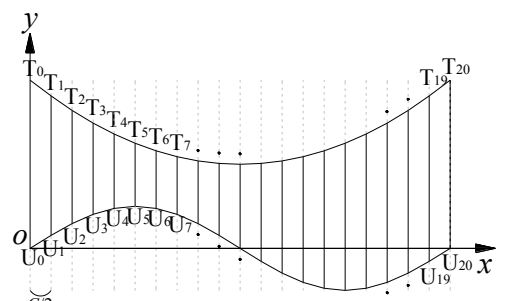

$C / 2$

(c)

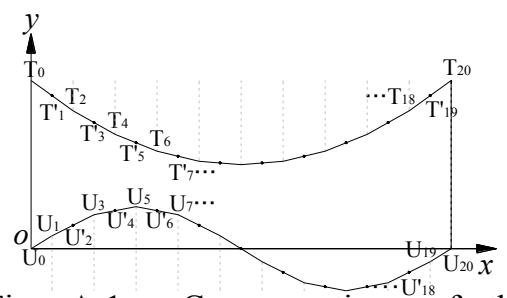

Fig. A-1 Cross sections of the honeycomb shown in Fig. 15. (a) trace the cross-section. (b) Discretization to dot sequence T, U. (c) Modification methods.
Table A-2 FLD parameters of Fig. 15 (mm).

\begin{tabular}{rrrrrrr}
$m$ & $a_{3 m-1}$ & $a_{3 m}$ & $a_{3 m+1}$ & $b_{3 m}$ & $b_{3 m+1}$ & $b_{3 m+2}$ \\
\hline 0 & - & 0.00 & 40.0 & 3.10 & 36.4 & 69.7 \\
\hline 1 & 40.0 & 67.2 & 94.4 & 69.7 & 91.6 & 114 \\
\hline 2 & 94.4 & 113 & 131 & 114 & 129 & 144 \\
\hline 3 & 131 & 145 & 159 & 144 & 158 & 172 \\
\hline 4 & 159 & 174 & 190 & 172 & 189 & 206 \\
\hline 5 & 190 & 210 & 230 & 206 & 230 & 253 \\
\hline 6 & 230 & 256 & 282 & 253 & 283 & 314 \\
\hline 7 & 282 & 315 & 347 & 314 & 349 & 384 \\
\hline 8 & 347 & 383 & 419 & 384 & 422 & 460 \\
\hline 9 & 419 & 458 & 496 & 460 & 450 & 539 \\
\hline 10 & 496 & 536 & 576 & 539 & 536 & - \\
\hline
\end{tabular}

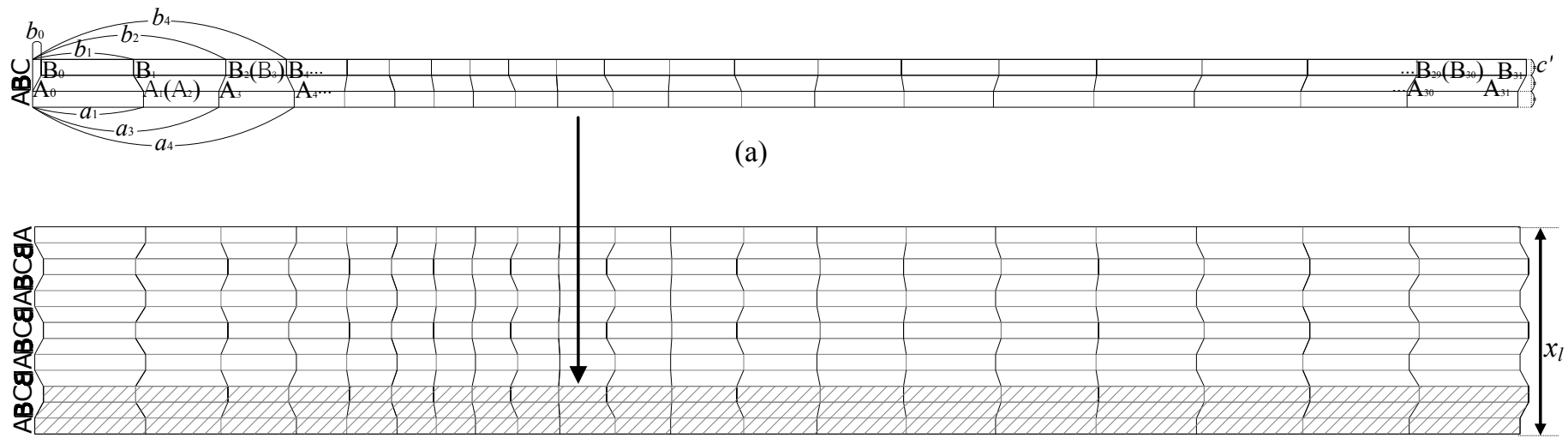

(b)

Fig. A-2 Drawing FLD from $a_{i}$ and $b_{i}$. 